\title{
Nonlinear Control and Synchronization with Time Delays of Multiagent Robotic Systems
}

\author{
Yassine Bouteraa, ${ }^{1,2}$ Jawhar Ghommam, ${ }^{1}$ Nabil Derbel, ${ }^{1}$ and Gérard Poisson ${ }^{2}$ \\ ${ }^{1}$ Research Unit on Intelligent Control, Design and Optimization of Complex Systems, National Engineering School of Sfax, \\ University of Sfax, BP W, Sfax 3038, Tunisia \\ ${ }^{2}$ Laboratoire Prisme - Pôle IRAuS, Université d’ Orléans, 63 avenue de Lattre de Tassigny 18020 Bourges Cedex, France
}

Correspondence should be addressed to Yassine Bouteraa, yassine.bouteraa@gmail.com

Received 3 January 2011; Accepted 20 April 2011

Academic Editor: Weizhou Su

Copyright ( $) 2011$ Yassine Bouteraa et al. This is an open access article distributed under the Creative Commons Attribution License, which permits unrestricted use, distribution, and reproduction in any medium, provided the original work is properly cited.

We investigate the cooperative control and global asymptotic synchronization Lagrangian system groups, such as industrial robots. The proposed control approach works to accomplish multirobot systems synchronization under an undirected connected communication topology. The control strategy is to synchronize each robot in position and velocity to others robots in the network with respect to the common desired trajectory. The cooperative robot network only requires local neighbor-to-neighbor information exchange between manipulators and does not assume the existence of an explicit leader in the team. It is assumed that network robots have the same number of joints and equivalent joint work spaces. A combination of the lyapunov-based technique and the cross-coupling method has been used to establish the multirobot system asymptotic stability. The developed control combines trajectory tracking and coordination algorithms. To address the time-delay problem in the cooperative network communication, the suggested synchronization control law is shown to synchronize multiple robots as well as to track given trajectory, taking into account the presence of the time delay. To this end, Krasovskii functional method has been used to deal with the delay-dependent stability problem.

\section{Introduction}

Nowadays, much research has been focusing on group coordination, cooperative control, and synchronization problems. In fact, motivated by the profit acquired by using multiple inexpensive systems working together to achieve complex tasks exceeding the abilities of a single agent, cooperative synchronization control has received significant attention. Distributed coordination and decentralized synchronization of multiagent systems have recently been studies extensively in the context of cooperative control [1-5], to name a few. In particular, design based on graph theory and Laplacian matrix produce interesting results [6-9]. Agreement, consensus problems in the area of cooperative control of multiagent systems have been studied in $[7,8,10-12]$. The coordination control strategies are closely related to the synchronization problem in which control laws are coupled and each agent robot control is updated using local rule based on its own sensors and the states of its neighbors. In this context, one recent representative work [13] shows that we can synchronize the multicomposed system in the case of partial knowledge, that is, only position measuring. A decentralized tracking control law globally exponentially synchronizes an arbitrary number of robots and represents a generalization of the average consensus problem. This has been presented in [5]. A synchronization approach to trajectory tracking of multiple mobile robots while maintaining time-varying formations has been presented in [14]. Adaptive control strategy to position synchronization of multiple motion axes using cross-coupling technology has been developed in [15]. In many engineering applications, communication delays between subsystems cannot be neglected. Therefore, the problem of time-delayed communication in control of multirobot systems is important in numerous practical applications. Indeed, without control measures of time delays in cooperative task may even cause instability. The 
problem of time-delayed communication in control of multiagent systems has been studied in several references $[7,16-$ 18]. The consideration of time-delayed communication in control of multirobot systems is a mainly practical necessity. In particular, this need occurs when addressing areas which require real-time applications such as operations in unsafe environments and robotic surgery.

The objective of this paper is to design a control approach that can achieve both synchronization of the robot movements and asymptotic stable tracking of a common desired trajectory. The proposed controller relies principally on a consensus algorithm for systems modeled by nonlinear second-order dynamics and applies the algorithm to the synchronization control problem by choosing appropriately information states on which consensus is reached. The concept key of the new synchronizing controller is the introduction of a state vector that quantifies the coordination degree between a robot manipulator positions and different positions of its neighbors. In the literature, most of earlier works on multiagent coordination and consensus $[3,4,7$, 19] mainly deal with very simple dynamic models such as linear systems and focuses on an algorithm taking the form of first-order dynamics $[11,20,21]$. In particular, most previous works on consensus and coordination of multiagent systems using the graph theory and laplacian [3, 4, 7-9] have presented a synchronization to the weighted average of initial conditions but they do not consider multiagent systems where there is a desired path to follow. Therefore, the aforementioned algorithms cannot give solutions for robot networks, where a desired trajectory is required. In contrast, the present work deals with highly nonlinear systems. Moreover, the developed approach achieves not only global asymptotic synchronization of the configuration variables, but also global asymptotic convergence to the desired trajectory. Notable works have focused on highly nonlinear systems. Their developed strategy requires the coupling feedback of the most adjacent robots [5] or axis [15] for the algorithm. However, the proposed strategy is based in partial mesh topology in which there are interconnections between all robots, such that all robots have direct influence in the combined dynamics. We provide by the use of partial mesh topology a high degree of reliability due to the presence of multiple paths for data between robots. On the other hand, it is not a fully connected mesh topology and consequently we avoid the expense and the complexity required for a connection between every robot in the network. In this paper, we study the problem of mutual synchronization when there are communication delays in the network. The delays are assumed to be bounded.

\section{Background and Preliminaries}

2.1. Modelling Multi-Lagrangian System Network. The $n$ degree-of-freedom robot manipulator composed of rigid bodies is expressed based on Newton's and Euler's equations as follows:

$$
M_{i}\left(q_{i}\right) \ddot{q}_{i}+C_{i}\left(q_{i}, \dot{q}_{i}\right) \dot{q}_{i}+g_{i}\left(q_{i}\right)=\tau_{i},
$$

where $q_{\mathrm{i}} \in \mathbb{R}^{n}$ denotes the joint angles of the $i$ th manipulator, $\dot{q}_{i} \in \mathbb{R}^{n}$, and $\ddot{q}_{i} \in \mathbb{R}^{n}$ are the vectors of joint velocity and joint acceleration, respectively. $M_{i}\left(q_{i}\right) \in \mathbb{R}^{n \times n}$ represents inertia matrix which is symmetric uniformly bounded and positive definite. $C_{i}\left(q_{i}, \dot{q}_{i}\right) \dot{q}_{i} \in \mathbb{R}^{n}$ is a vector function containing Coriolis and centrifugal forces. $g_{i}\left(q_{i}\right) \in \mathbb{R}^{n}$ is a vector function consisting of gravitational forces. Although the above equations of motion are coupled and nonlinear, they exhibit certain fundamental properties due to their Lagrangian dynamic structure. The most important property is the well-known skew symmetry of the matrix $\dot{M}-2 C$ [22].

2.2. Multiagent Communication Topology. Since we based on our coordination algorithm conception on consensus strategy and concepts of graph theory, we present several basic properties of these technology. Let $G=(V, E)$ a digraph with $N$ nodes, the set of nodes $V=1,2, \ldots, n$, and edges $E \subseteq V \times V$. Each node is labeled by $v_{i} \in V$ and each edge is denoted by $e_{i j}=\left(v_{i}, v_{j}\right)$. Neighbors of agent $v_{i}$ are denoted by $N=\left\{v_{j} \in V /\left(v_{i}, v_{j}\right) \in E\right\}$. The adjacency matrix $A=\left[a_{i j}\right] \in \mathbb{R}^{n \times n}$ of a weighted digraph is defined as

$$
\begin{gathered}
a_{i j}>0 \quad \text { if }\left(v_{i}, v_{j}\right) \in E, \\
a_{i j}=0, \quad \text { elsewhere. }
\end{gathered}
$$

Agent $i$ communicates with agent $j$ if $j$ is a neighbor of $i$ or if $a_{i j} \neq 0$. Note that an edge $e_{i j}$ in a directed graph means that robot $j$ can reach information from robot $i$, but not necessarily vice versa. In contrast, in an undirected graph, pairs of node are unordered and an edge $e_{i j}$ implies that robots $i$ and $j$ can get information from one another. The adjacency matrix of an undirected graph has the same meaning as that of the directed graph except that $a_{i j}=a_{j i}$. The degree matrix of the digraph $G=(V, E)$ is a diagonal matrix defined as

$$
d_{i j}= \begin{cases}\operatorname{deg}_{\text {out }}\left(v_{i}\right) & \text { if }(i=j) \in E, \\ 0, & \text { otherwise, }\end{cases}
$$

where $\operatorname{deg}_{\text {out }}\left(v_{i}\right)=\sum_{j=1}^{n} a_{i j}$.

The graph laplacian of $G$ is defined as $L=D-A$, where $D=\left[d_{i i}\right]$ the degree matrix of $G$. In the undirected graph case, $L$ is symmetric positive semidefinite. In the present topology, the edge represents bidirectional communication links. This consists on a group of $n$ manipulators interchanging information that can be viewed as an undirected graph (see Figure 1).

\section{Controller Design}

3.1. Tracking and Synchronization Errors. In this paper, we consider the synchronization of multiple robots following a common time-varying trajectory. We will design decentralized control laws for $n$ robots manipulators such that all joint positions mutually synchronize and track a common desired trajectory. The control objective of the proposed synchronization controller scheme is to synchronize the 


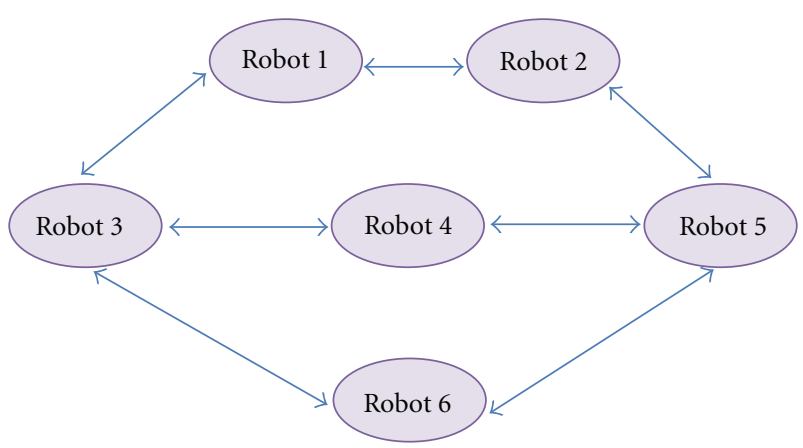

FIgURE 1: Undirected graph topology structure.

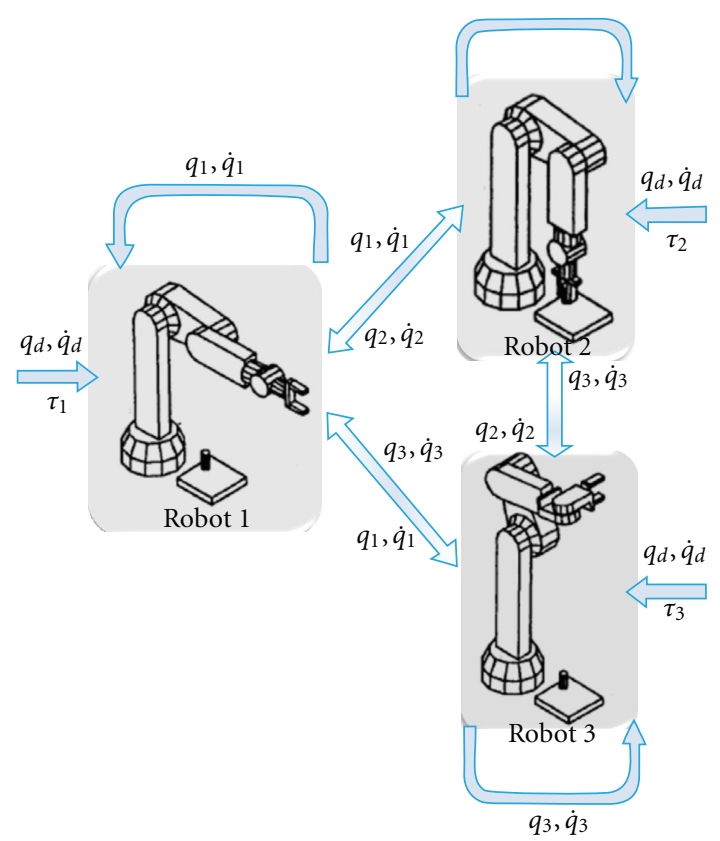

FIGURE 2: Robot network using bidirectional communication.

$i$ th joint position and velocity $q_{i}, \dot{q}_{i}$ to the state of any manipulator $q_{j}, \dot{q}_{j}$. Besides the controller is required to regulate the joint positions $q_{j}$ to track a desired trajectory $q_{d}$. Specifically, the control torque for the $i$ th robot is to control the tracking error to converge to zero and at the same time, to synchronize motions of $n$ robots in communication so that the synchronization error converges to zero. To this end, we define the measure of the position tracking error of the $i$ th manipulator as

$$
e_{1 i}(t)=q_{i}(t)-q_{d}(t)+\int_{t_{0}}^{t} \Lambda_{i}\left[q_{i}(\lambda)-q_{d}(\lambda)\right] d \lambda,
$$

where $\Lambda_{i}$ is a diagonal positive definite matrix. Information on the vector $e_{1 i}$ will give insight on the convergence of the joint positions to the desired trajectory. It is required to know the performance of the controller that is to know how the trajectory of each robot manipulator converges with respect to each other. There are various ways to choose the synchronization error. For example in [13], authors include the error information of all systems involved in

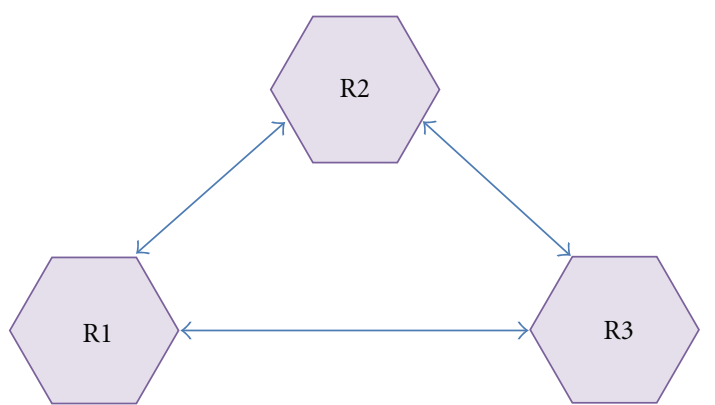

Figure 3: The topology model of the robots in simulation.

the synchronization. Our approach will make use of the cross-coupling technique to propose a feasible and efficient synchronization error, which consists on a measure of the synchronization for robot manipulator as defined as follows:

$$
e_{2 i}(t)=\sum_{j \neq i} \beta_{i j}\left(q_{i}-q_{j}\right)
$$

where, $\beta_{i j}$ is a diagonal positive definite matrix which gives insight on the weighted communication among the robot network.

3.2. Feedback Control Design. The objective of this paper is to design individual tracking controller for $n$ manipulators such that they coordinate their motions and track synchronously a desired trajectory. To this end, we define the global error which encompasses both synchronization error and trajectory tracking error for manipulator $i$ as

$$
e_{i}=e_{1 i}+\int_{t_{0}}^{t} e_{2 i}(\lambda) d \lambda
$$

Under the above strategy, motions of all manipulators are synchronized. The control of each manipulator considers motion responses of the other manipulators for synchronization. It takes into account only robots which make the exchange of information with it. The objective is to design a control law such that the coupling errors, that is, the position errors, velocity errors, and synchronization errors, all converge to zero. For each manipulator, the control law $\tau_{i}$ is defined as follows:

$$
\begin{aligned}
\tau_{i}= & C_{i}\left(q_{i}, \dot{q}_{i}\right) \dot{q}_{i}+g_{i}\left(q_{i}\right) \\
& +M_{i}\left(q_{i}\right)\left[\ddot{q}_{d}-K_{p i} e_{i}-K_{d i} \dot{e}_{i}-\Lambda_{i}\left(\dot{q}_{i}-\dot{q}_{d}\right)\right] \\
& +M_{i}\left(q_{i}\right)\left[\sum_{j \neq i} K_{i j}\left(e_{2 i}-e_{2 j}\right)+\Lambda_{i} e_{2 i}\right],
\end{aligned}
$$

where $q_{d}$ is a common trajectory reference to be tracked, which is a smooth time-varying trajectory and for which the first and the second derivatives exist for all $t \geq 0$. $K_{d i}$ is a symmetric positive definite matrix. $K_{i j}$ is a matrix that reflects the quality of communication channels; it is a symmetric positive matrix. 


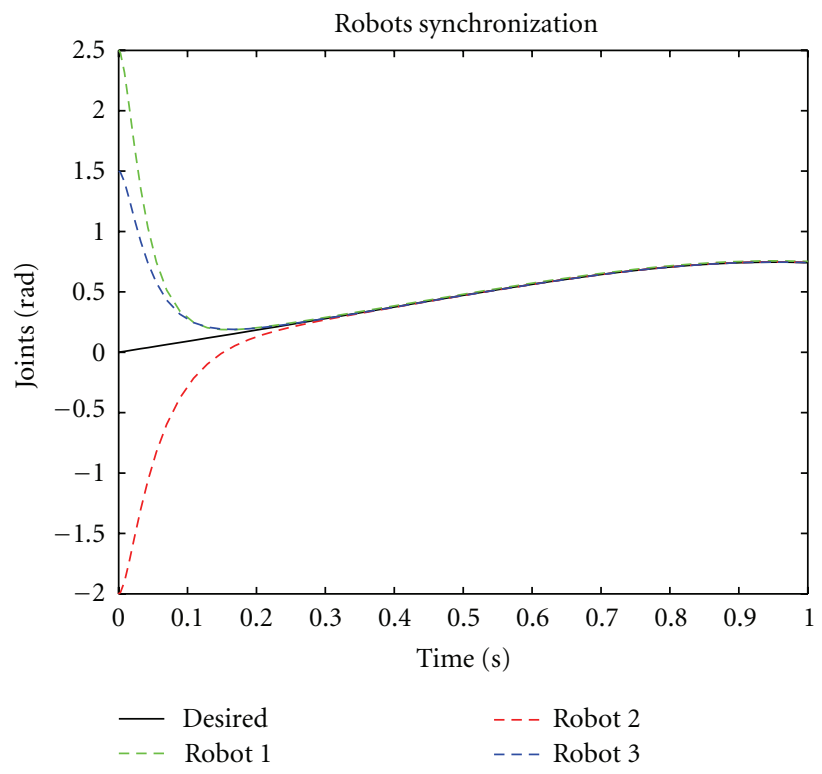

(a)

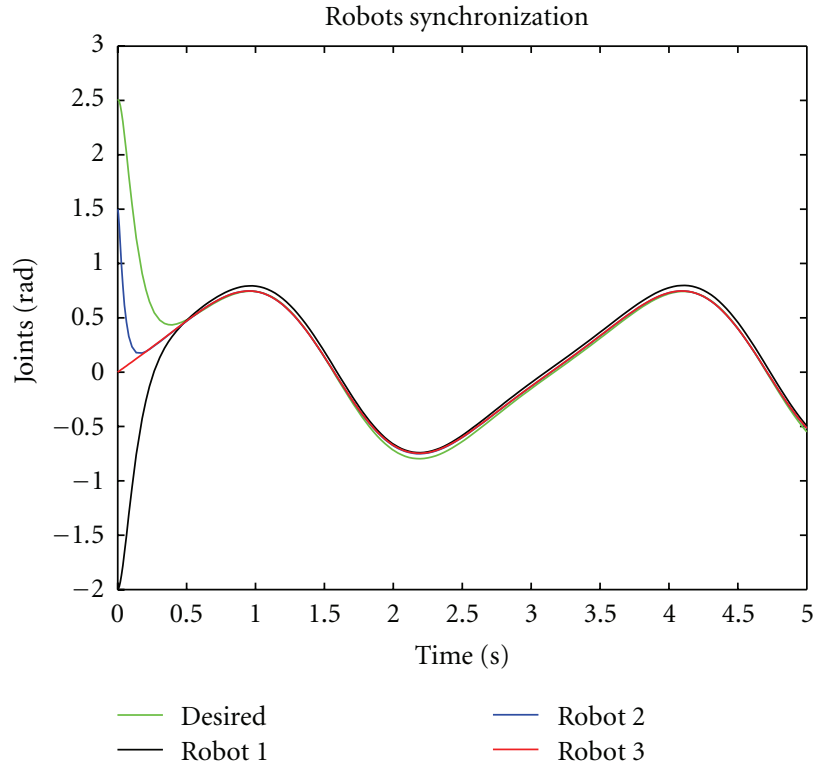

(b)

FIGURE 4: Robots synchronization and trajectory tracking.

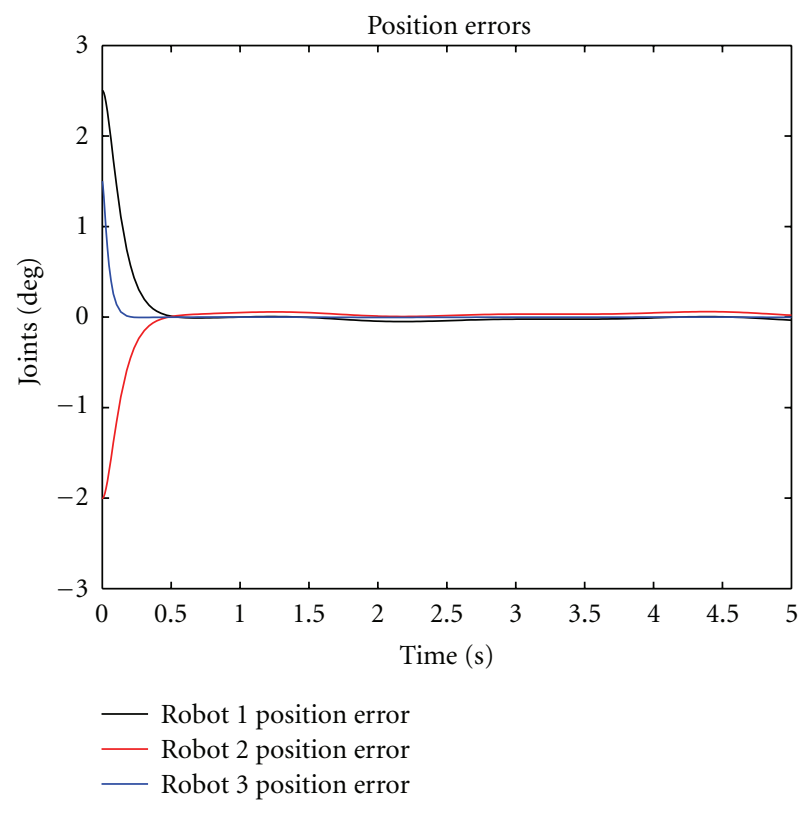

Figure 5: Position errors.

3.3. Stability Analysis. Substituting (7) into (1) yields

$$
\begin{aligned}
M_{i}\left(q_{i}\right) \ddot{q}_{i}= & M_{i}\left(q_{i}\right)\left[\ddot{q}_{d}-K_{p i} e_{i}-K_{d i} \dot{e}_{i}-\Lambda_{i}\left(\dot{q}_{i}-\dot{q}_{d}\right)\right] \\
& +M_{i}\left(q_{i}\right)\left[\sum_{j \neq i} K_{i j}\left(e_{2 i}-e_{2 j}\right)+\Lambda_{i} e_{2 i}\right]
\end{aligned}
$$

This results in

$$
\begin{aligned}
\ddot{q}_{i}= & \ddot{q}_{d}-K_{p i} e_{i}-K_{d i} \dot{e}_{i}-\Lambda_{i}\left(\dot{q}_{i}-\dot{q}_{d}\right) \\
& +\sum_{j \neq i} K_{i j}\left(e_{2 i}-e_{2 j}\right)+\Lambda_{i} e_{2 i},
\end{aligned}
$$

which can be written as follows:

$$
\ddot{e}_{i}=-K_{p i} e_{i}-K_{d i} \dot{e}_{i}+\sum_{j \neq i} K_{i j}\left(e_{2 i}-e_{2 j}\right)+\Lambda_{i} e_{2 i}+\dot{e_{2 i}} \text {. }
$$

Using the expression of the synchronization error $e_{2 i}$ and its first derivative gives

$$
\begin{aligned}
\ddot{e}_{i}= & -K_{p i} e_{i}-K_{d i} \dot{e}_{i}+\sum_{j \neq i} K_{i j}\left(e_{2 i}-e_{2 j}\right) \\
& +\sum_{j \neq i}\left[K_{i j}\left(\dot{q}_{i}-\dot{q}_{d}\right)-\left(\dot{q}_{j}-\dot{q}_{d}\right)\right] \\
& +\sum_{j \neq i} \Lambda_{i} K_{i j}\left[\left(q_{i}-q_{d}\right)-\left(q_{j}-q_{d}\right)\right] .
\end{aligned}
$$

Further calculation, will result in

$$
\ddot{e}_{i}=-K_{p i} e_{i}-K_{d i} \dot{e}_{i}+\sum_{j \neq i} K_{i j}\left(\dot{e}_{i}-\dot{e}_{j}\right) .
$$

Equation (12) represents the closed loop synchronized system for the $i$ th manipulator. In the sequel we proceed to analyze the stability properties of the proposed synchronized control scheme and ultimately to show that control goals: the position errors, velocity errors, and synchronization errors, all converge to zero. To prove the stability of the overall synchronized system, we define

$$
e^{T}=\left[e_{1}^{T}, e_{2}^{T}, \ldots, e_{n}^{T}\right]^{T}
$$


Using (12) we obtain the synchronized error dynamics

$$
\ddot{e}=-K_{p} e-K_{d} \dot{e}+K_{c} \dot{e},
$$

where $K_{p}=\operatorname{diag}\left(K_{p i}\right), K_{d}=\operatorname{diag}\left(K_{d i}\right)$ and $K_{c}$ is given by

$$
\left(\begin{array}{ccccc}
\sum_{j \neq 1} K_{1 j} & \cdots & -K_{1 j} & \cdots & -K_{1 n} \\
\cdots & \cdots & \cdots & \cdots & \cdots \\
-K_{i 1} & \cdots & \sum_{j \neq i} K_{i j} & \cdots & -K_{i n} \\
\cdots & \cdots & \cdots & \cdots & \cdots \\
-K_{n 1} & \cdots & -K_{n j} & \cdots & \sum_{j \neq n} K_{n j}
\end{array}\right) .
$$

Note that $K_{c}$ is symmetric and positive semidefinite matrix, since we have an undirected graph, that is, $K_{i j}=K_{j i}$.

The synchronized error dynamics (14) is a linear time invariant system described by a second-order linear differential equation. A sufficient condition for the error dynamics to be stable is that the matrices $K_{p}$ and $K_{d}-K_{c}$ are positive definite. In particular, matrices $K_{d i}$ can be diagonal satisfying

$$
K_{d i}>2 \sum_{i \neq j} K_{i j}
$$

To analyze the stability properties of the closed-loop error dynamics (19), we take the following definite and radially unbounded Lyapunov function candidate:

$$
V=\dot{e}^{T} \dot{e}+e^{T} K_{p} e .
$$

Its derivative to respect to time is

$$
\dot{V}=-\dot{e}^{T}\left(K_{d}-K_{c}\right), \quad \dot{e} \leq 0 .
$$

It follows by direct application of Lasalle's invariance principle that the origin $(e, \dot{e})=(0,0)$ is globally asymptotically stable and $\lim \dot{e} \rightarrow 0$ for $t \rightarrow \infty$.

Referring to the expression of the global error (6):

$$
\begin{aligned}
e_{i}= & q_{i}-q_{d}+\int_{t_{0}}^{t} \Lambda\left[q_{i}(\lambda)-q_{d}(\lambda)\right] d \lambda \\
& +\int_{t_{0}}^{t} \sum_{j \neq i} \beta_{i j}\left(q_{i}-q_{j}\right)
\end{aligned}
$$

as $\dot{e}=0$ we have

$$
\dot{q}_{i}-\dot{q}_{d}=-\Lambda_{i}\left(q_{i}-q_{d}\right)-\sum_{j \neq i} \beta_{i j}\left(q_{i}-q_{j}\right) .
$$

Setting $\varepsilon_{i}=q_{i}-q_{d}$, then (20) can be written as

$$
\dot{\varepsilon}_{i}=-\Lambda_{i} \varepsilon_{i}-\sum_{j \neq i} \beta_{i j}\left(\varepsilon_{i}-\varepsilon_{j}\right) .
$$

Our objective is to show that $\lim \varepsilon_{i}=0$ for $t \rightarrow \infty$. To this end, we define $\varepsilon=\left[\varepsilon_{1} \cdots \cdots \varepsilon_{n}\right]^{T}$ and $\Lambda=$ $\left[\Lambda_{1} \cdots \cdots \cdots \Lambda_{n}\right]^{T}$. Then $(21)$ can be written as

$$
\dot{\varepsilon}=A \cdot \varepsilon,
$$

where matrix $\mathrm{A}$ is given by

$$
\left(\begin{array}{ccccc}
-\Lambda_{1}-\sum_{j \neq 1} \beta_{1 j} & \cdots & \beta_{1 j} & \cdots & \beta_{1 n} \\
\cdots & \cdots & \cdots & \cdots & \cdots \\
\beta_{i 1} & \cdots & -\Lambda_{i}-\sum_{j \neq i} \beta_{i j} & \cdots & \beta_{i n} \\
\cdots & \cdots & \cdots & \cdots & \cdots \\
\beta_{n 1} & \cdots & \beta_{n j} & \cdots & -\Lambda_{n}-\sum_{j \neq n} \beta_{n j}
\end{array}\right) .
$$

We set the Lyapunov function candidate as

$$
v(t)=\varepsilon^{T} \mathcal{E} .
$$

Differentiating $v(t)$ with respect to time yields

$$
\begin{aligned}
& \dot{v}=2 \sum_{i=1}^{n} \varepsilon_{i} \dot{\varepsilon}_{i}, \\
& \dot{v}=2 \sum_{i=1}^{n} \varepsilon_{i}\left(-\Lambda_{i} \varepsilon_{i}-\sum_{j \neq i} \beta_{i j}\left(\varepsilon_{i}-\varepsilon_{j}\right)\right), \\
& \dot{v}=-2 \sum_{i=1}^{n} \Lambda_{i} \varepsilon_{i}^{2}-2 \sum_{i=1}^{n} \sum_{j \neq i} \beta_{i j}\left(\varepsilon_{i}-\varepsilon_{j}\right) \varepsilon_{i}, \\
& \dot{v}=-2 \sum_{i=1}^{n} \Lambda_{i} \varepsilon_{i}^{2}-2 \sum_{i=1}^{n} \sum_{j \neq i} \beta_{i j} \varepsilon_{i}^{2}+2 \sum_{i=1}^{n} \sum_{j \neq i} \beta_{i j} \varepsilon_{i} \varepsilon_{j},
\end{aligned}
$$

knowing that

$$
\sum_{i=1}^{n} \sum_{j \neq i} \beta_{i j} \varepsilon_{i}^{2}=\sum_{j=1}^{n} \sum_{i \neq j} \beta_{j i} \varepsilon_{j}^{2}
$$

Consequently,

$$
\dot{v}=-2 \sum_{i=1}^{n} \Lambda_{i} \varepsilon_{i}^{2}-\sum_{i=1}^{n} \sum_{j \neq i} \beta_{i j}\left(\varepsilon_{i}-\varepsilon_{j}\right)^{2} \leq 0 .
$$

It follows by direct application of Lasalle's invariance that the origin is globally asymptotically stable. Consequently we obtain $\lim \varepsilon_{i}(t) \rightarrow 0$ for $t \rightarrow \infty$. Then $q_{i} \rightarrow q_{d}$ and $\dot{q}_{i} \rightarrow \dot{q}_{d}$ for $t \rightarrow \infty$.

Referring to (20), we show that $q_{i} \rightarrow q_{j}$ for $t \rightarrow \infty$.

\section{Coordination with Time Delays}

In this section, we study the coordination control problem taking into account time delays of communication channels. As a first assumption, we suppose that these delays can be justified by the fact that data information sent by the neighboring vehicles $j \neq i$ reaches vehicle $i$ after a timedelay due to the short-range communication channels. To take into account the time delay produced during the communication among the robots, we introduced in a coordination error expression a term $\tau$ which represents the same time delay due to the short-range communication channels. Therefore, 


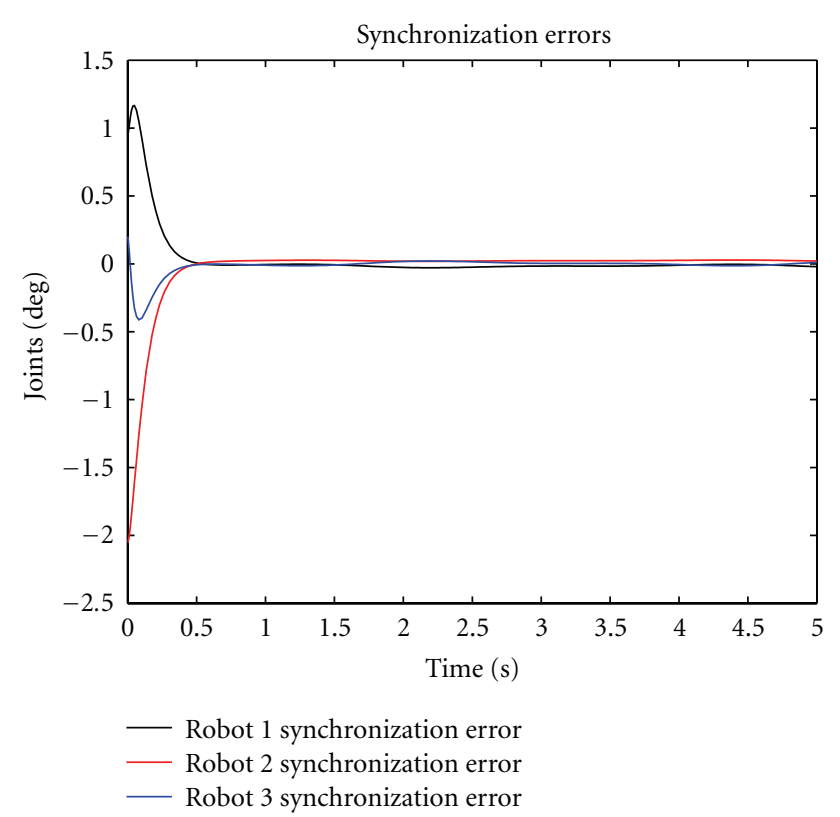

Figure 6: Synchronization errors.

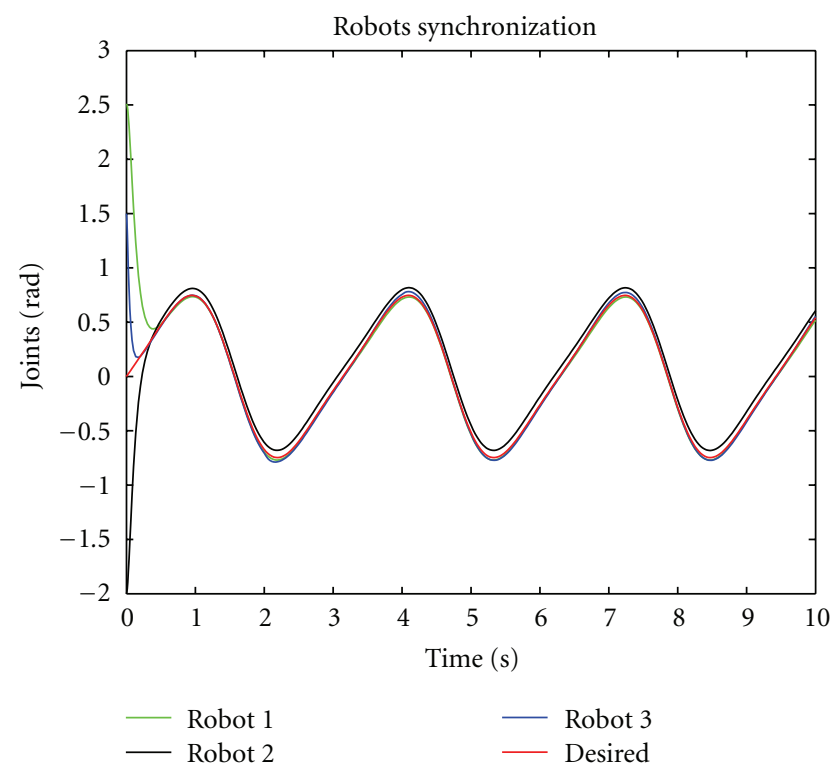

FIGURE 7: Robots synchronization without communication delay.

a coordination error, in the time delay context, will be presented as the well-known classical time delayed model of multiagent network:

$$
e_{2 i}(t)=\sum_{j \neq i} \beta_{i j}\left[q_{i}(t-\tau)-q_{j}(t-\tau)\right]
$$

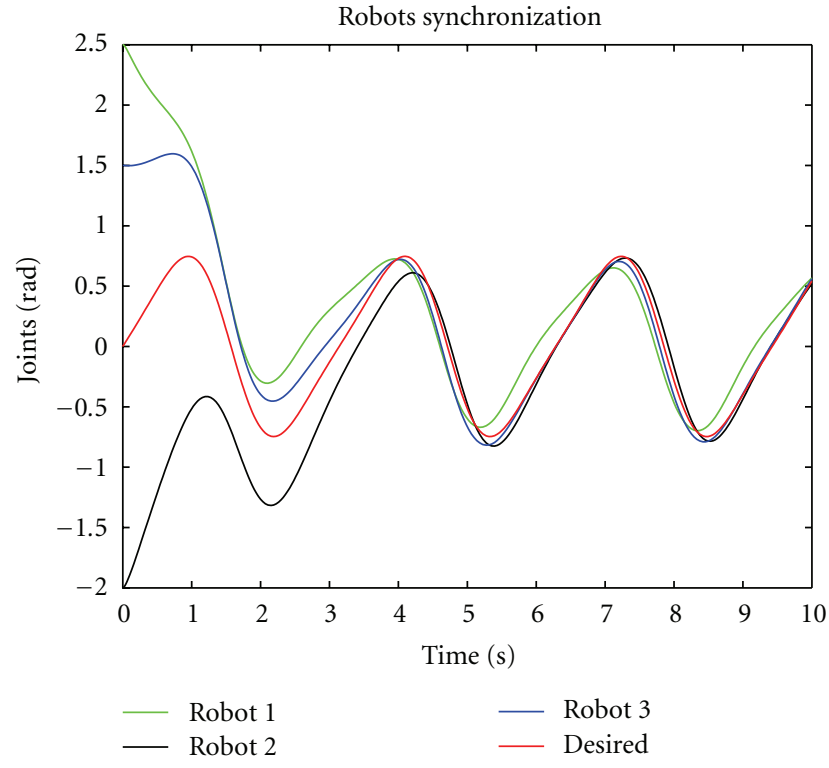

FIGURE 8: Robots synchronization in presence of time delay $(0.5 \mathrm{~s})$.

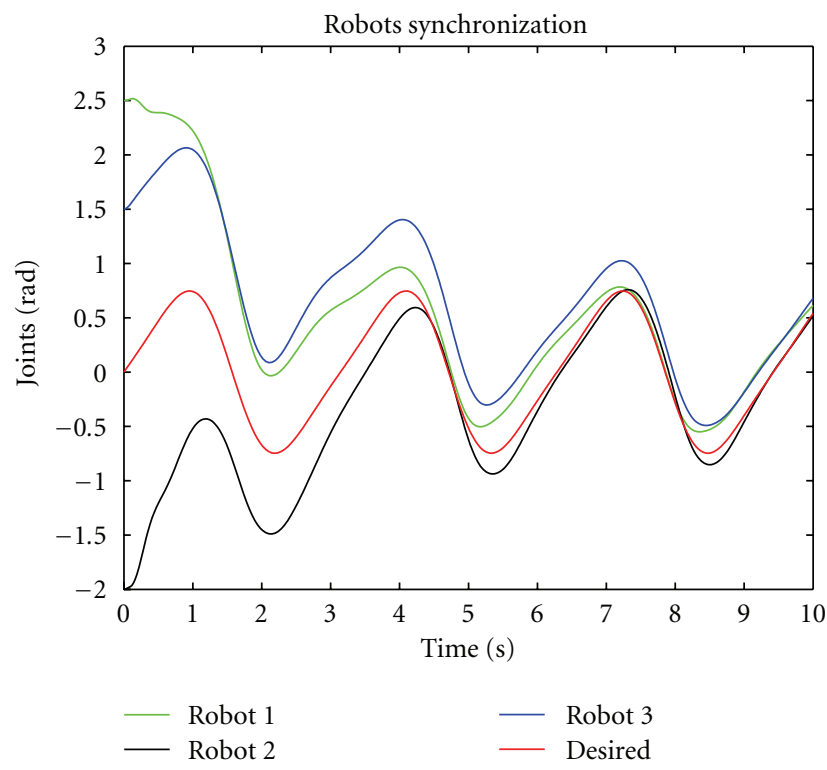

FIGURE 9: Robots synchronization in presence of time delay (1 s).

Consequently, the controller implanted in each lagrangian system among the network take the following expression:

$$
\begin{aligned}
\tau_{i}= & C_{i}\left(q_{i}, \dot{q}_{i}\right) \dot{q}_{i}+g_{i}\left(q_{i}\right) \\
& +M_{i}\left(q_{i}\right)\left[\ddot{q}_{d}-K_{p i} e_{i}(t)-K_{d i} \dot{e}_{i}(t)-\Lambda_{i}\left(\dot{q}_{i}-\dot{q}_{d}\right)\right] \\
& +M_{i}\left(q_{i}\right)\left[\sum_{j \neq i} K_{i j}\left[e_{2 i}(t-\tau)-e_{2 j}(t-\tau)\right]+\Lambda_{i} e_{2 i}(t-\tau)\right] .
\end{aligned}
$$

It will be shown that the behavior of the coordinated system under the effect of time delay changes significantly. 


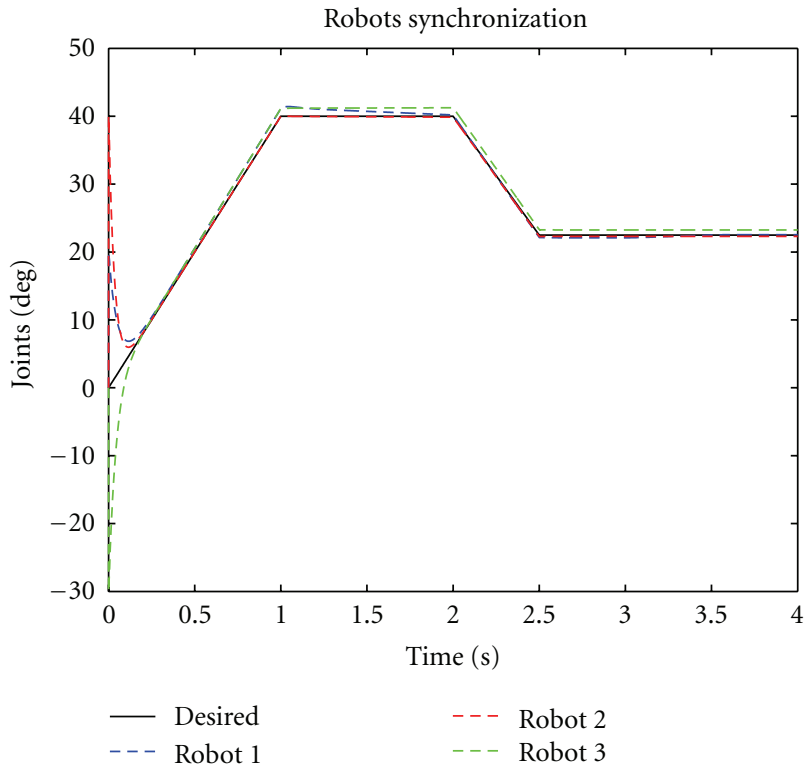

Figure 10: Robots synchronization without time delay.

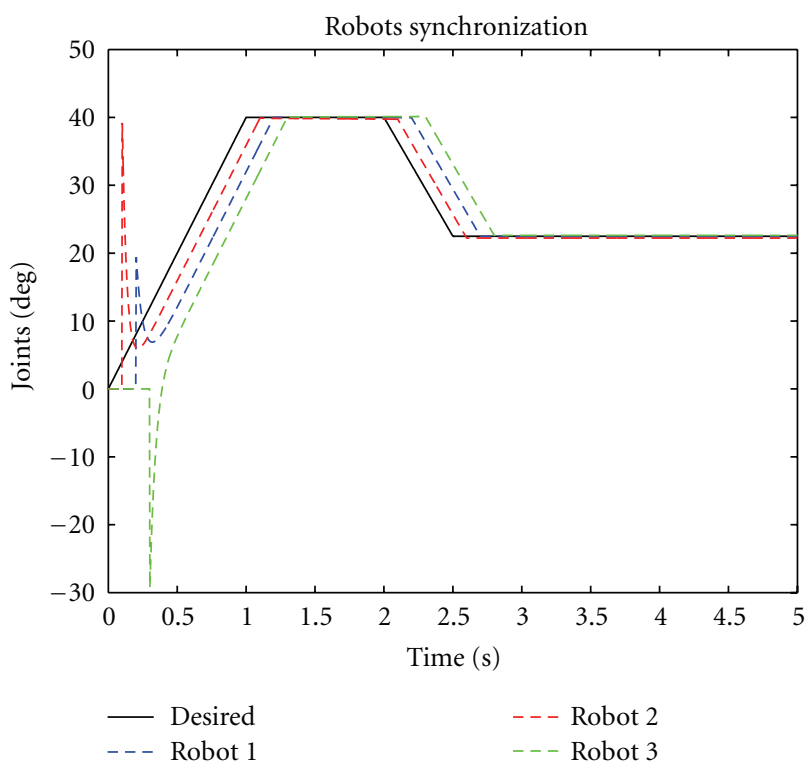

FIGURE 11: Robots synchronization in presence of time delay.

4.1. Stability Analysis. Substituting (29) into (1) yields

$M_{i}\left(q_{i}\right) \ddot{q}_{i}$

$$
\begin{aligned}
= & M_{i}\left(q_{i}\right)\left[\ddot{q_{d}}-K_{p i} e_{i}-K_{d i} \dot{e}_{i}-\Lambda_{i}\left(\dot{q}_{i}-\dot{q}_{d}\right)\right] \\
& +M_{i}\left(q_{i}\right)\left[\sum_{j \neq i} K_{i j}\left[e_{2 i}(t-\tau)-e_{2 j}(t-\tau)\right]+\Lambda_{i} e_{2 i}(t-\tau)\right] .
\end{aligned}
$$

Multiplying by $M^{-1}$ and adding $\dot{e}_{2 i}(t-\tau)$ in both sides yields

$$
\begin{aligned}
\ddot{q}_{i}- & \ddot{q}_{d}+\Lambda_{i}\left(\dot{q}_{i}-\dot{q}_{d}\right)+\dot{e}_{2 i}(t-\tau) \\
= & -K_{p i} e_{i}-K_{d i} \dot{e}_{i} \\
& +\sum_{j \neq i} K_{i j}\left[e_{2 i}(t-\tau)-e_{2 j}(t-\tau)\right] \\
& +\Lambda_{i} e_{2 i}(t-\tau)+\dot{e}_{2 i}(t-\tau) .
\end{aligned}
$$

Using the expression of the synchronization error $e_{2 i}$ and its first derivative gives

$$
\begin{aligned}
\ddot{e}_{i}= & -K_{p i} e_{i}-K_{d i} \dot{e}_{i}+\sum_{j \neq i} K_{i j}\left[e_{2 i}(t-\tau)-e_{2 j}(t-\tau)\right] \\
& +\sum_{j \neq i} K_{i j}\left[\left(\dot{q}_{i}-\dot{q}_{d}\right)-\left(\dot{q}_{j}-\dot{q}_{d}\right)\right] \\
& +\sum_{j \neq i} \Lambda_{i} K_{i j}\left[\left(q_{i}-q_{d}\right)-\left(q_{j}-q_{d}\right)\right] .
\end{aligned}
$$

Further calculation, we obtain the synchronized error dynamics

$$
\ddot{e}=-K_{p} e-K_{d} \dot{e}+K_{c} \dot{e}(t-\tau),
$$

where $K_{p}, K_{d}$, and $K_{c}$ are the same matrices already defined (see Section 3.3). By the Leibnitz formula, we have

$$
\dot{e}-\dot{e}(t-\tau)=\int_{t-\tau}^{t} \ddot{e}(\lambda) d \lambda
$$

substituting (34) into (33) leads to

$$
\ddot{e}=-K_{p} e-K_{d} \dot{e}+K_{c}\left(\dot{e}-\int_{t-\tau}^{t} \ddot{e}(\lambda) d \lambda\right) .
$$

Setting $\tilde{e}=[e, \dot{e}]^{T}$. Therefore (35) can be written as

$$
\dot{\tilde{e}}=\left(\begin{array}{cc}
0 & I \\
-K_{p} & -K_{d}+K_{c}
\end{array}\right) \tilde{e}-\left(\begin{array}{cc}
0 & 0 \\
0 & K_{c}
\end{array}\right) \int_{t-\tau}^{t} \dot{\tilde{e}}(\lambda) d \lambda,
$$

This yields the following form:

$$
\dot{\tilde{e}}=\beta_{0} \tilde{e}-\beta_{1} \int_{t-\tau}^{t} \dot{\tilde{e}}(\lambda) d \lambda
$$

with $\beta_{0}=\left(\begin{array}{cc}0 & I \\ -K_{p} & -K_{d}+K_{c}\end{array}\right)$ and $\beta_{1}=\left(\begin{array}{ll}0 & 0 \\ 0 & K_{c}\end{array}\right)$.

To analyze the stability of the global system, we consider the following Lyapunov-Krasovskii functional (LKF):

$$
\begin{aligned}
v(t) & =v_{1}(t)+v_{2}(t)+v_{3}(t), \\
v_{1}(t) & =\widetilde{e}^{T}(t) P \widetilde{e}(t), \\
v_{2}(t) & =\int_{t-\tau}^{t} \widetilde{e}^{T}(\lambda) R \widetilde{e}(\lambda) d \lambda, \\
v_{3}(t) & =\int_{-\tau}^{0} \int_{t+s}^{t} \dot{\tilde{e}}^{T}(\alpha) Z \dot{\widetilde{e}}(\alpha) d \alpha d \lambda,
\end{aligned}
$$


where $P=P^{T}>0 ; R=R^{T}>0 ; Z=Z^{T}>0$ are weighting matrices of appropriate dimensions. A straightforward computation gives the time derivative of $v(\mathrm{t})$ along the solution of (37) as

$$
\begin{aligned}
\dot{v}(t)= & \delta^{T}\left[2 N^{T} P M+N^{T} R N-Q^{T} R Q+\tau M^{T} Z M\right] \delta \\
& -\int_{t-\tau}^{t} \dot{\widetilde{e}}^{T}(\lambda) Z \dot{\widetilde{e}}(\lambda) d \lambda,
\end{aligned}
$$

where $\delta=\left[\tilde{e}^{T}(t), \tilde{e}^{T}(t-\tau)\right]^{T} ; N=\left[\beta_{0}, \beta_{1}\right] ; M=[I, 0] ; Q=$ $[0, I]$. The Jensen's inequality gives a suitable bound for the last term of (39).

$$
\begin{aligned}
-\int_{t-\tau}^{t} \dot{\tilde{e}}^{T}(\lambda) Z \dot{\tilde{e}}(\lambda) d \lambda & \leq \int_{t-\tau}^{t} \dot{\tilde{e}}^{T}(\lambda) d \lambda\left(\frac{Z}{\tau}\right) \int_{t-\tau}^{t} \dot{\tilde{e}}(\lambda) d \lambda \\
& \leq-\tilde{e}^{T} T^{T}\left(\frac{Z}{\tau}\right) T \tilde{e}
\end{aligned}
$$

with $T=[I,-I]$. The time derivative of the LKF (38) can thus be bounded by $\dot{v}(t) \leq \delta^{T} \xi \delta$, where $\xi=2 N^{T} P M+$ $N^{T} R N-Q^{T} R Q+\tau M^{T} Z M-\left(1 / \tau T^{T} Z T\right)$. Then if the LMI $\xi<0$ is satisfied, the derivative of the Lyapunov-Krasovskii functional is negative definite. To ensure that matrix $\xi<0$ is negative definite, we select appropriate control gains $K_{p}>$ $K_{p}^{*}$ and $K_{d}-K_{c}>K^{*}$ through processing Matlab's LMI solver such that

$$
2 N^{T} P M+N^{T} R N-Q^{T} R Q+\tau M^{T} Z M-\frac{1}{\tau T^{T} Z T}<0 .
$$

Then, if the LMI $\xi<0$ is satisfied, the derivative of the Lyapunov-Krasovskii functional is therefore negative definite. In consequence the origin $\tilde{e}=0$ is asymptotically stable.

This results in $e \rightarrow 0$ for $t \rightarrow \infty$ and $\dot{e} \rightarrow 0$ for $t \rightarrow \infty$. The proof for asymptotic convergence of the coordinated tracking error $\tilde{e}$ is not sufficient to prove the convergence to zero of both error $e_{1}$ and $e_{2}$. Our concern now is to show that coordination is successfully realized for a specific time delay $\tau_{c}$.

The proof pursued the same line reasoning as the proof of Section 3.3. Consequently, we obtain the following equation derived from the global error expression:

$$
\dot{\varepsilon}_{i}=-\Lambda_{i} \varepsilon_{i}-\sum_{j \neq i} K_{i j}\left(\varepsilon_{i}(t-\tau)-\varepsilon_{j}(t-\tau)\right) .
$$

Rewriting all states of (42) into a compact representation and applying the Laplace transform leads to

$$
s \mathcal{\varepsilon}(s)-\varepsilon(0)=-\Lambda \mathcal{\varepsilon}(s)-e^{-\tau s} K_{c} \mathcal{E}(s) .
$$

This can be written as

$$
\mathcal{E}(s)=\left(s I+\Lambda+e^{-\tau s} K_{c}\right)^{-1} \mathcal{E}(0) .
$$

If the characteristic equation $P(s, \tau)$ : det $\left|s I+\Lambda+e^{-\tau s} K_{c}\right|=0$ has all its zeros in the left half complex plan then the system is stable and one can easily conclude about the convergence of $q_{i}$ to $q_{d}$. Since the ordinal system, free from time delay
TABLE 1: Control gains.

\begin{tabular}{lccc}
\hline $\begin{array}{l}\text { Control Gains } \\
\text { and JIC }\end{array}$ & Robot 1 & Robot 2 & Robot 3 \\
\hline$k_{d}$ & $\operatorname{diag}\{20\}$ & $\operatorname{diag}\{15\}$ & $\operatorname{diag}\{25\}$ \\
$k_{p}$ & $\operatorname{diag}\{18\}$ & $\operatorname{diag}\{10\}$ & $\operatorname{diag}\{15\}$ \\
$k_{12}$ & $\operatorname{diag}\{0.8\}$ & $\operatorname{diag}\{0.8\}$ & \\
$k_{13}$ & $\operatorname{diag}\{0.5\}$ & & $\operatorname{diag}\{0.5\}$ \\
$k_{23}$ & $\operatorname{diag}\{0.2\}$ & $\operatorname{diag}\{0.2\}$ & \\
JIC & $(2.5,2.5)$ & $(-2,-2)$ & $(1.5,1.5)$ \\
\hline
\end{tabular}

(i.e., $\tau=0)$ is stable and that $P(s, \tau)$ is a continuous function of $\tau$, then using the D-Decomposition, the minimal positive solution to the following equation:

$$
\tau_{c_{i}}=\frac{\arccos \left(-\Lambda_{i} / K_{c i}\right)}{\sqrt{\left(K_{c i^{2}}-\Lambda_{i}^{2}\right)}}
$$

would make all the zeros of the characteristic equation in the left half complex plane. Therefore if we select $\tau \in\left[0, \tau_{c}\right]$, where $\tau c=\sup \left(\tau_{c_{i}}\right)$ for all $1 \leq i \leq n$, solutions of (44) converge to zero and consequently $q_{i} \rightarrow q_{d}$ for $t \rightarrow \infty$, $\dot{q}_{i} \rightarrow \dot{q}_{d}$ for $t \rightarrow \infty$, and $q_{i} \rightarrow q_{j}$ for $t \rightarrow \infty$.

\section{Simulation Results}

To show the effectiveness of the proposed synchronizing controller we provide some simulation results. These simulations were proposed for a network of 3 identical robot manipulators interconnected under a cooperative scheme as shown in Figure 2.

Let the communication structure among the robots described by an undirected strongly connected graph topology as shown in Figure 3. We set Joint Initial Conditions (JICs), coupling and control gains for the three robots as discussed below (see Table 1). Simulations are performed on Matlab/Simulink. Figure 4 illustrates the synchronization of robots that follow a common trajectory. This proves that the tracking and synchronization objectives are attained by the proposed controller.

Figures 5 and 6, show, respectively, the convergence of error positions to zero and the convergence of synchronizing errors to zero, explaining how robots, while tracking the desired trajectory, synchronize their positions. The effect of time delays on the coordination of robots is shown in the following write-up. First, the delay-free case is presented in Figure 7 in which it is shown how the three angular positions asymptotically synchronize. Next, we consider the time delay in communication. Synchronization while tracking periodic trajectory is shown in Figures 8 and 9. From these figures, it is seen that the robots do not have the same starting positions. The speed for achieving an agreement depends essentially on the time-delay communication channels. Figures 10 and 11 illustrate that the behavior of the coordinated system changes significantly, under the effect of time delay. 


\section{Conclusion}

This paper has considered the synchronization problem in distributed multi-Lagrangian systems. The aim of this work was to find out a decentralized controller, which individually applied to each lagrangian system, the synchronization in position and velocity is therefore met. Reaching synchronization stability of highly nonlinear robot dynamics constitutes one of the main contributions of this paper. The proposed control law ensures the robots' states synchronization while tracking a common desired trajectory. Another aspect of robots coordination and trajectory tracking control was investigated. In the coordination strategy there are practically interconnections between all the systems, such that all systems have influence on the overall dynamics. The proposed algorithm works under cooperative scheme in the sense that it does not require any explicit leaders in the team. The studied topology is connected under an undirected interaction graph. To deal with time-delay problem in communication between robots, the proposed decentralized control guarantees that the information variables of each robot reach agreement even in the presence of communication delay. Illustrative examples have shown the effectiveness of the described strategy. Future work will address the coordination control of under actuated lagrangian systems.

\section{References}

[1] W. Ren and Y. Cao, "Overview of recent research in distributed multi-agent coordination," in Distributed Coordination of Multi-agent Networks: Communications and Control Engineering, pp. 23-41, Springer, New York, NY, USA, 2011.

[2] J. Ghommam, H. Mehrjerdi, M. Saad, and F. Mnif, "Formation path following control of unicycle-type mobile robots," Robotics and Autonomous Systems, vol. 58, no. 5, pp. 727-736, 2010.

[3] A. Jadbabaie, J. Lin, and A. S. Morse, "Coordination of groups of mobile autonomous agents using nearest neighbor rules," IEEE Transactions on Automatic Control, vol. 48, no. 6, pp. 988-1001, 2003.

[4] Z. Lin, M. Broucke, and B. Francis, "Local control strategies for groups of mobile autonomous agents," IEEE Transactions on Automatic Control, vol. 49, no. 4, pp. 622-629, 2004.

[5] S. J. Chung and J.-J. E. Slotine, "Cooperative robot control and concurrent synchronization of lagrangian systems," IEEE Transactions on Robotics, vol. 25, no. 3, pp. 686-700, 2009.

[6] M. Mesbahi and F. Y. Hadaegh, "Formation flying control of multiple spacecraft via graphs, matrix inequalities, and switching," Journal of Guidance, Control, and Dynamics, vol. 24, no. 2, pp. 369-377, 2001.

[7] R. Olfati-Saber and R. M. Murray, "Consensus problems in networks of agents with switching topology and time-delays," IEEE Transactions on Automatic Control, vol. 49, no. 9, pp. 1520-1533, 2004.

[8] W. Ren, "Multi-vehicle consensus with a time-varying reference state," Systems and Control Letters, vol. 56, no. 7-8, pp. 474-483, 2007.

[9] W. Ren, W. Beard, and E. Atkins, "Information consensus in multivehicle cooperative control: collective group behavior through local interaction," IEEE Control Systems Magazine, vol. 27, no. 2, pp. 71-82, 2007.
[10] B. Lei and L. Zeng, "Consensus algorithm for swarm robotics cooperative control," Advanced Materials Research, vol. 201203, pp. 1854-1857, 2011.

[11] W. Ren and R. W. Beard, "Consensus seeking in multiagent systems under dynamically changing interaction topologies," IEEE Transactions on Automatic Control, vol. 50, no. 5, pp. 655-661, 2005.

[12] J.-J. E. Slotine and W. Wang, "A study of synchronization and group cooperation using partial contraction theory," in Cooperative Control, V. Kumar, N. E. Leonard, and A. S. Morse, Eds., vol. 309 of Lecture Notes in Control and Information Sciences, pp. 443-446, Springer, New York, NY, USA, 2004.

[13] A. Rodriguez-Angeles and H. Nijmeijer, "Mutual synchronization of robots via estimated: state feedback: a cooperative approach," IEEE Transactions on Control Systems Technology, vol. 12, no. 4, pp. 542-554, 2004.

[14] D. Sun, C. Wang, W. Shang, and G. Feng, "A synchronization approach to trajectory tracking of multiple mobile robots while maintaining time-varying formations," IEEE Transactions on Robotics, vol. 25, no. 5, pp. 1074-1086, 2009.

[15] D. Sun, "Position synchronization of multiple motion axes with adaptive coupling control," Automatica, vol. 39, no. 6, pp. 997-1005, 2003.

[16] N. Chopra and M. W. Spong, "Output synchronization of nonlinear systems with time delay in "communication"', in Proceedings of the 45th IEEE Conference on Decision and Control, San Diego, Calif, USA, December 2006.

[17] W. Wang and J.-J. E. Slotine, "Contraction analysis of timedelayed communications and group cooperation," IEEE Transactions on Automatic Control, vol. 51, no. 4, pp. 712-717, 2006.

[18] H. G. Tanner and D. K. Christodoulakis, "State synchronization in local-interaction networks is robust with respect to time delays," in Proceedings of the 44th IEEE Conference on Decision and Control, pp. 4945-4950, Sevilla, Spain, December 2005.

[19] Z. Qu, J. Wang, and R. A. Hull, "Cooperative control of dynamical systems with application to autonomous vehicles," IEEE Transactions on Automatic Control, vol. 53, no. 4, pp. 894-911, 2008.

[20] J. R. Lawton and R. W. Beard, "Synchronized multiple spacecraft rotations," Automatica, vol. 38, no. 8, pp. 13591364, 2002.

[21] R. Olfati-Saber, J. A. Fax, and R. M. Murray, "Consensus and cooperation in networked multi-agent systems," Proceedings of the IEEE, vol. 95, no. 1, Article ID 4118472, pp. 215-233, 2007.

[22] M. W. Spong and N. Chopra, "Synchronization of networked lagrangian systems," in Lagrangian and Hamiltonian Methods for Nonlinear Control 2006, F. Bullo and K. Fujimoto, Eds., vol. 366 of Lecture Notes in Control and Information Sciences, pp. 47-59, Springer, New York, NY, USA, 2007. 

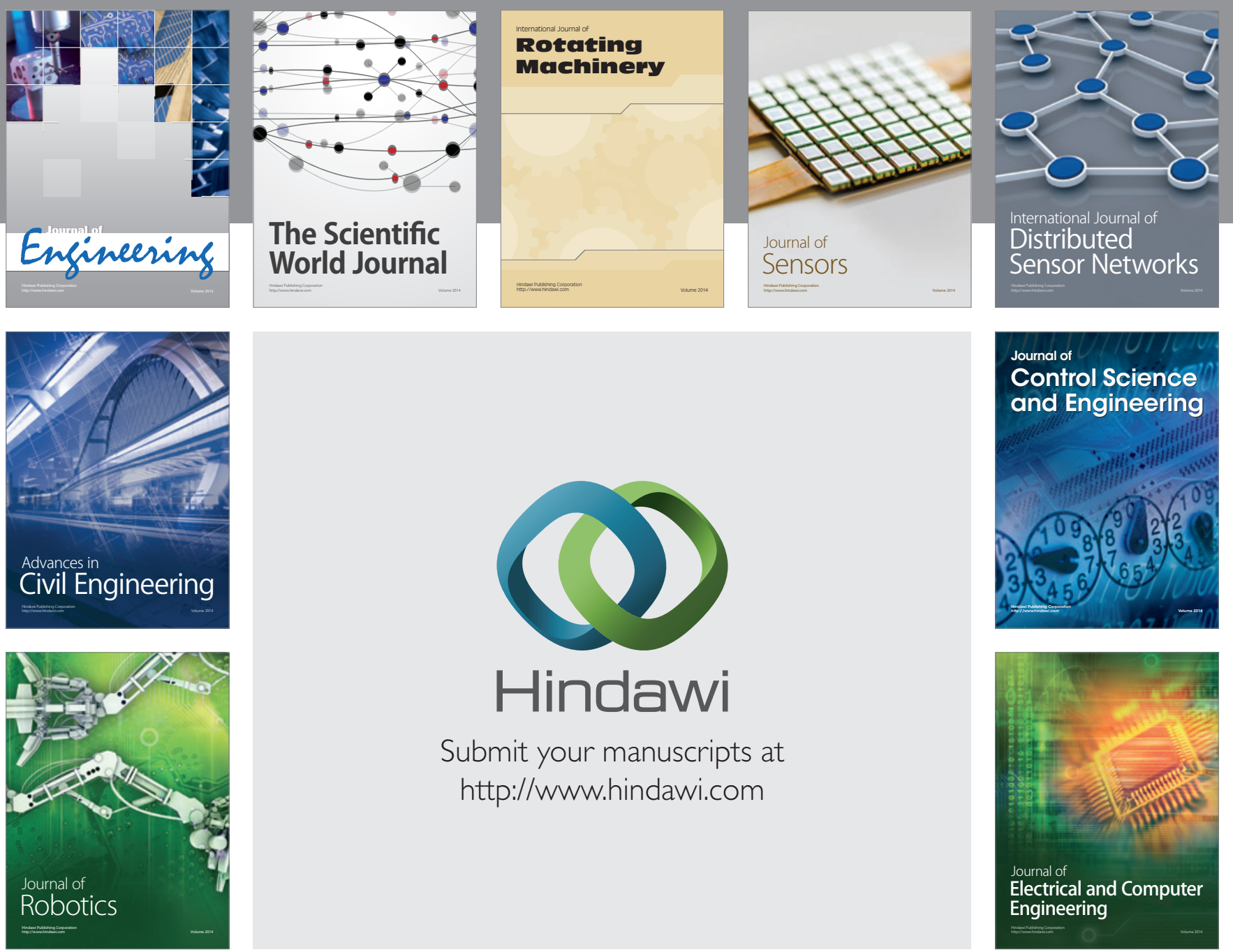

Submit your manuscripts at

http://www.hindawi.com
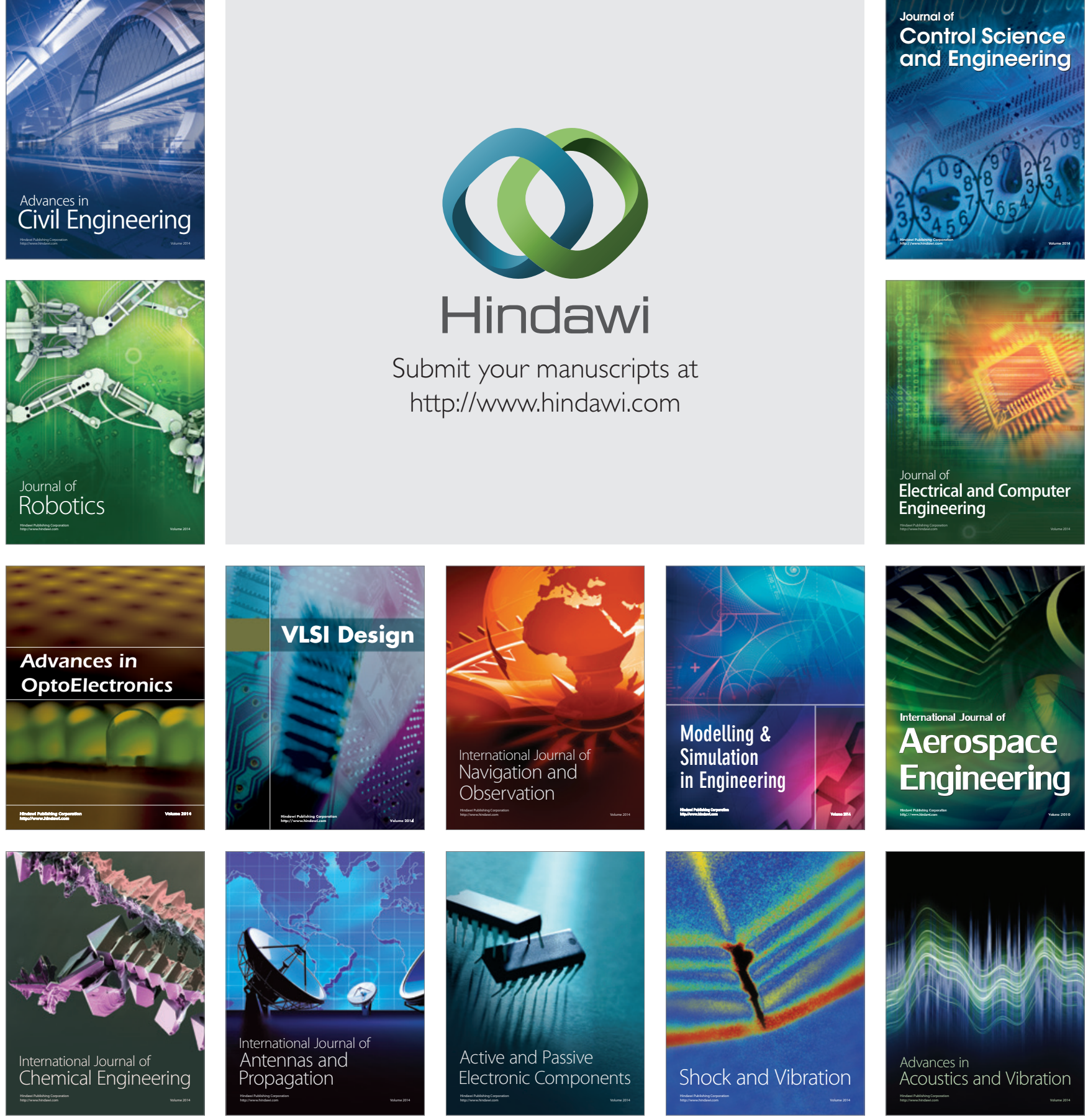